\title{
A randomized trial of bevacizumab for newly diagnosed glioblastoma.
}

\author{
Mark R Gilbert \\ Department of Neuro-Oncology, University of Texas M.D. Anderson Cancer Center
}

James J Dignam

American College of Radiology, Philadelphia; University of Chicago

Terri S Armstrong

Department of Neuro-Oncology, University of Texas M.D. Anderson Cancer Center; University of Texas Health Science Center School of Nursing

5elfpry thişandepdditional works at: https://jdc.jefferson.edu/radoncfp

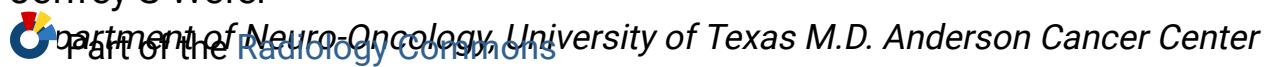

betotals kinewshow access to this document benefits you

Tel-Aviv Medical Center

\section{Recommended Citation}

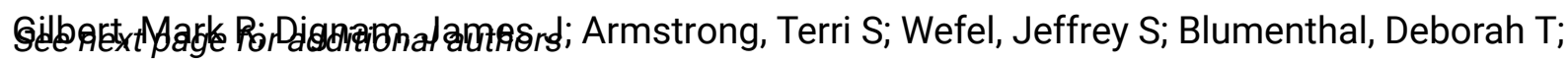
Vogelbaum, Michael A; Colman, Howard; Chakravarti, Arnab; Pugh, Stephanie; Won, Minhee; Jeraj, Robert; Brown, Paul D; Jaeckle, Kurt A; Schiff, David; Stieber, Volker W; Brachman, David G; Werner-Wasik, Maria; Tremont-Lukats, Ivo W; Sulman, Erik P; Aldape, Kenneth D; Curran, Walter J; and Mehta, Minesh P, "A randomized trial of bevacizumab for newly diagnosed glioblastoma." (2014). Department of Radiation Oncology Faculty Papers. Paper 47.

https://jdc.jefferson.edu/radoncfp/47

This Article is brought to you for free and open access by the Jefferson Digital Commons. The Jefferson Digital Commons is a service of Thomas Jefferson University's Center for Teaching and Learning (CTL). The Commons is a showcase for Jefferson books and journals, peer-reviewed scholarly publications, unique historical collections from the University archives, and teaching tools. The Jefferson Digital Commons allows researchers and interested readers anywhere in the world to learn about and keep up to date with Jefferson scholarship. This article has been accepted for inclusion in Department of Radiation Oncology Faculty Papers by an authorized administrator of the Jefferson Digital Commons. For more information, please contact: JeffersonDigitalCommons@jefferson.edu. 


\section{Authors}

Mark R Gilbert, James J Dignam, Terri S Armstrong, Jeffrey S Wefel, Deborah T Blumenthal, Michael A Vogelbaum, Howard Colman, Arnab Chakravarti, Stephanie Pugh, Minhee Won, Robert Jeraj, Paul D Brown, Kurt A Jaeckle, David Schiff, Volker W Stieber, David G Brachman, Maria Werner-Wasik, Ivo W Tremont-Lukats, Erik P Sulman, Kenneth D Aldape, Walter J Curran, and Minesh P Mehta 


\title{
The NEW ENGLAN D \\ JOURNAL of MEDICINE
}

ESTABLISHED IN 1812

FEBRUARY 20, 2014

VOL. 370 NO. 8

\section{A Randomized Trial of Bevacizumab for Newly Diagnosed Glioblastoma}

\author{
Mark R. Gilbert, M.D., James J. Dignam, Ph.D., Terri S. Armstrong, Ph.D., A.N.P.-B.C., Jeffrey S. Wefel, Ph.D., \\ Deborah T. Blumenthal, M.D., Michael A. Vogelbaum, M.D., Ph.D., Howard Colman, M.D., Ph.D., \\ Arnab Chakravarti, M.D., Stephanie Pugh, Ph.D., Minhee Won, M.A., Robert Jeraj, Ph.D., Paul D. Brown, M.D., \\ Kurt A. Jaeckle, M.D., David Schiff, M.D., Volker W. Stieber, M.D., David G. Brachman, M.D., \\ Maria Werner-Wasik, M.D., Ivo W. Tremont-Lukats, M.D., Erik P. Sulman, M.D., Kenneth D. Aldape, M.D., \\ Walter J. Curran, Jr., M.D., and Minesh P. Mehta, M.D.
}

ABSTRACT

\section{BACKGROUND}

Concurrent treatment with temozolomide and radiotherapy followed by maintenance temozolomide is the standard of care for patients with newly diagnosed glioblastoma. Bevacizumab, a humanized monoclonal antibody against vascular endothelial growth factor A, is currently approved for recurrent glioblastoma. Whether the addition of bevacizumab would improve survival among patients with newly diagnosed glioblastoma is not known.

\section{METHODS}

In this randomized, double-blind, placebo-controlled trial, we treated adults who had centrally confirmed glioblastoma with radiotherapy (60 Gy) and daily temozolomide. Treatment with bevacizumab or placebo began during week 4 of radiotherapy and was continued for up to 12 cycles of maintenance chemotherapy. At disease progression, the assigned treatment was revealed, and bevacizumab therapy could be initiated or continued. The trial was designed to detect a $25 \%$ reduction in the risk of death and a $30 \%$ reduction in the risk of progression or death, the two coprimary end points, with the addition of bevacizumab.

\section{RESULTS}

A total of 978 patients were registered, and 637 underwent randomization. There was no significant difference in the duration of overall survival between the bevacizumab group and the placebo group (median, 15.7 and 16.1 months, respectively; hazard ratio for death in the bevacizumab group, 1.13). Progression-free survival was longer in the bevacizumab group (10.7 months vs. 7.3 months; hazard ratio for progression or death, 0.79 ). There were modest increases in rates of hypertension, thromboembolic events, intestinal perforation, and neutropenia in the bevacizumab group. Over time, an increased symptom burden, a worse quality of life, and a decline in neurocognitive function were more frequent in the bevacizumab group.

\section{CONCLUSIONS}

First-line use of bevacizumab did not improve overall survival in patients with newly diagnosed glioblastoma. Progression-free survival was prolonged but did not reach the prespecified improvement target. (Funded by the National Cancer Institute; ClinicalTrials.gov number, NCT00884741.)
From the University of Texas M.D. Anderson Cancer Center (M.R.G., T.S.A., J.S.W., P.D.B., I.W.T.-L., E.P.S., K.D.A.) and the University of Texas Health Science Center School of Nursing (T.S.A.), Houston; American College of Radiology (J.J.D., S.P., M.W.) and Thomas Jefferson University (M.W.-W.) - both in Philadelphia; the University of Chicago, Chicago (J.J.D.); Tel-Aviv Medical Center, Tel Aviv, Israel (D.T.B.); Cleveland Clinic, Cleveland (M.A.V.); the University of Utah, Salt Lake City (H.C.); Ohio State University, Columbus (A.C.); University of Wisconsin, Madison (R.J.); Mayo Clinic, Jacksonville, FL (K.A.J.); University of Virginia, Charlottesville (D.S.); Southeast Cancer Control Consortium, Winston-Salem, NC (V.W.S.); Barrow Neurologic Institute, Phoenix, AZ (D.G.B.); Emory University, Atlanta (W.J.C.); and the University of Maryland, Baltimore (M.P.M.). Address reprint requests to $D r$. Gilbert at the Department of Neuro-Oncology, University of Texas M.D. Anderson Cancer Center, 1515 Holcombe Blvd., Unit 431, Houston, TX 77030.

N EnglJ Med 2014;370:699-708 DOI: 10.1056/NEJMoal308573

Copyright (๑) 2014 Massachusetts Medical Society. 
LIOBLASTOMA IS THE MOST COMMON primary malignant brain tumor in adults. After maximal surgical tumor resection, the current standard of care is based on a phase 3, randomized clinical trial conducted by the European Organization for Research and Treatment of Cancer and the National Cancer Institute of Canada, which showed that concurrent treatment with daily temozolomide and radiotherapy followed by maintenance temozolomide was superior to radiotherapy alone. ${ }^{1,2}$

Despite the improvement in outcomes with this combined chemoradiotherapy approach, few patients survive beyond 5 years; therefore, new therapeutic strategies are needed. ${ }^{3}$ Angiogenesis is a prominent feature of glioblastoma, most commonly attributed to the autocrine and paracrine production of vascular endothelial growth factor $A$ (VEGF-A), which up-regulates the VEGF signaltransduction pathway. ${ }^{4,5}$ Several approaches have been used to target this prominent component of the tumor biology. Small-molecule tyrosine kinase inhibitors of this pathway, such as cediranib and sorafenib, have shown minimal efficacy. ${ }^{6,7}$ Bevacizumab is a humanized monoclonal antibody against the VEGF-A ligand that binds to its circulating target, altering the kinetics of ligand binding to endothelial cells and down-regulating angiogenesis. ${ }^{8}$ Initial studies that explored the efficacy of bevacizumab in adults with recurrent glioblastoma showed clinical activity, including a reduction in tumor size, a prolongation of progression-free survival, and an overall lowering of glucocorticoid requirements to control tumor-related edema.9,10 These results led to the accelerated Food and Drug Administration approval of bevacizumab for patients with recurrent glioblastoma.

Preclinical models suggest that antiangiogenic therapies cause temporary vascular normalization, leading to improved blood flow, which in turn should improve the delivery of oxygen and chemotherapeutic agents, potentially enhancing the efficacy of both radiotherapy and chemotherapy. ${ }^{11}$ As a collaborative effort of the Radiation Therapy Oncology Group (RTOG), the North Central Cancer Treatment Group (NCCTG), and the Eastern Cooperative Oncology Group (ECOG), we conducted a randomized, placebo-controlled, doubleblind, phase 3 trial, called RTOG 0825, to test the hypothesis that antiangiogenic therapy improves the efficacy of the standard chemoradiotherapy for glioblastoma.

\section{METHODS}

\section{STUDY PATIENTS}

Patients were eligible for the study if they were at least 18 years of age and had newly diagnosed glioblastoma (World Health Organization [WHO] grade IV astrocytoma), as confirmed on central review. Additional eligibility criteria included a Karnofsky performance status of at least 70 (on a scale from 0 to 100 , with higher numbers indicating a higher activity level) and adequate hematologic, renal, and hepatic function (Table S1 in the Supplementary Appendix, available with the full text of this article at NEJM.org). Patients with active cardiac disease or recent cerebrovascular events were excluded. In addition, patients were required to undergo an imaging study (computed tomography [CT] or magnetic resonance imaging [MRI]) performed within 1 week before study registration) to rule out recent intracranial hemorrhage. Patients who were receiving glucocorticoids had to have received a stable or decreasing dose for the 5 days before study registration. Also required was the submission of a paraffinembedded tumor-tissue block with a minimum of $1 \mathrm{~cm}^{2}$ of tumor surface area before the initiation of radiotherapy.

All patients provided written informed consent. The study was approved by the institutional review board or the equivalent panel at each study center before patient enrollment.

\section{STUDY TREATMENT}

Patients had to be registered before the start of the concomitant radiotherapy and chemotherapy (for details, see Fig. S1 in the Supplementary Appendix). Fractionated, conformal radiotherapy or intensity-modulated radiotherapy (IMRT) was given at a daily dose of 2 Gy. Treatment was delivered 5 days a week for 6 weeks, for a total dose of $60 \mathrm{~Gy}$. Conformal therapy was delivered to an initial volume consisting of the area of enhancement, the postoperative cavity plus surrounding edema (or other abnormality as seen on fluidattenuated inversion recovery [FLAIR] images on MRI), and a 2-cm margin, for a total dose of $46 \mathrm{~Gy}$ in 23 fractions, followed by a boost of 14 Gy in 7 fractions to the area of enhancement plus the cavity and a $2.5-\mathrm{cm}$ margin. IMRT was permitted within protocol-defined guidelines at institutions that fulfilled IMRT-specific quality requirements, and all patients underwent radiotherapy quality assurance with the use of pre- 
defined guidelines. Treatment with temozolomide, at a dose of $75 \mathrm{mg}$ per square meter of bodysurface area, was started at the initiation of radiotherapy and was continued daily until the completion of radiotherapy, with a maximum of 49 doses.

Patients were randomly assigned to receive either bevacizumab or placebo in a permutedblock design. ${ }^{12}$ Stratification factors were status with respect to O-6-methylguanine-DNA methyltransferase (MGMT) and a tumor-based molecular profile based on expression of nine genes. ${ }^{13}$ MGMT status was determined with the use of a quantitative methylation-specific polymerase-chainreaction (PCR) assay performed centrally by OncoMethylome Sciences. ${ }^{14}$ The nine-gene assay was performed with the use of a PCR technique optimized for paraffin-embedded tumor samples, and results were dichotomized as either favorable or unfavorable. ${ }^{13}$

Bevacizumab (or placebo) was administered intravenously at a dose of $10 \mathrm{mg}$ per kilogram of body weight every 2 weeks, starting at week 4 of radiotherapy, until disease progression, severe treatment-related toxicity, or completion of adjuvant therapy (maximum number of doses, 24 over 12 cycles).

Maintenance treatment with temozolomide began 4 weeks after the completion of radiotherapy at a starting dose of $150 \mathrm{mg}$ per square meter for 5 consecutive days of a 28-day cycle, with an increase to $200 \mathrm{mg}$ per square meter for subsequent cycles if no treatment-related adverse events of grade 2 or higher were noted. Treatment was planned for 6 cycles with the option of extension to a total of 12 cycles if there were no or only low-grade adverse events and there was evidence of continued benefit. Antiemetic therapy with the use of a 5-hydroxytryptamine receptor antagonist was strongly recommended. Pneumocystis prophylaxis was recommended for patients with CD4 counts of less than 200 per cubic millimeter. At the time of tumor progression, patients could be informed about their study-group assignment and either begin or continue a bevacizumabcontaining regimen provided as part of the study.

\section{PATIENT EVALUATION AND FOLLOW-UP}

At baseline, all the patients underwent a physical examination that included a neurologic assessment, complete blood counts, blood chemical analyses (including tests of renal and hepatic function), and tumor imaging with either MRI (preferred) or CT, as well as a serum pregnancy test in women of child-bearing age. Patients were invited to participate in a longitudinal evaluation of the net clinical benefits of the treatment (NCB substudy) with the use of the M.D. Anderson Symptom Inventory-Brain Tumor Module (MDASI-BT), a neurocognitive-function test battery (Hopkins Verbal Learning Test-Revised [HVLT-R], Trail Making Test [TMT], and Controlled Oral Word Association [COWA]), and the European Organization for Research and Treatment of Cancer qualityof-life questionnaire with a brain-cancer module (EORTC QLQ-C30/BN20). ${ }^{15-18}$ Patients were administered the NCB substudy measures at the time of imaging studies. During radiotherapy, patients were assessed for adverse events weekly and underwent weekly complete blood counts and monthly blood chemical analyses. During the maintenance phase of treatment, patients underwent blood counts and blood chemical analyses on days 21 and 28 of each cycle.

A repeat tumor-imaging study was performed approximately 4 weeks after completion of radiotherapy and then before the initiation of cycle 4 of maintenance treatment (as well as before the initiation of cycles 7 and 10, if administered). Patients who completed adjuvant treatment underwent tumor imaging every 3 months until tumor progression. Response was assessed with the use of serial measures of the product of the two largest cross-sectional diameters, and progression was defined as an increase in tumor size by at least $25 \%$ or the development of a new lesion. ${ }^{19}$ Since early reactions to radiotherapy may emulate tumor progression, investigators were encouraged not to declare tumor progression within the first 12 weeks after completion of radiotherapy unless there was a new lesion or neurologic worsening. ${ }^{20}$ Toxic effects were recorded and graded according to the National Cancer Institute Common Terminology Criteria for Adverse Events (CTCAE), version 3.0.

\section{PRIMARY END POINTS}

The coprimary end points were the duration of overall survival from randomization, which was defined as the time until death from any cause, and the duration of progression-free survival, which was defined as the time until either disease progression or death.

\section{STUDY OVERSIGHT}

The trial, which was sponsored by the National Cancer Institute (which also provided the study 
drug), was developed by the first and last authors in collaboration with the RTOG Brain Committee, the RTOG Statistical Group, the Cancer Therapy Evaluation Program at the National Cancer Institute, the NCCTG, and the ECOG. An unrestricted educational grant for support of the study was provided by Genentech, which had no role in the collection of data, analysis of findings, or preparation of this report. All treatment data were collected by the RTOG data center and reviewed by the first author. The analyses were performed by RTOG statisticians. Central review was performed on all pathological specimens. The first draft of the manuscript was written by the first author with support from all coauthors; all authors reviewed and approved the manuscript. No one who is not an author contributed to the preparation of the manuscript. All the authors vouch for the completeness and accuracy of the data and confirm that the study was conducted according to the protocol, which is available at NEJM.org.

\section{STATISTICAL ANALYSIS}

The trial was designed to concurrently provide a power of $80 \%$ for the detection of a $25 \%$ relative reduction in the risk of death (hazard ratio, 0.75) and a $30 \%$ relative reduction in the risk of either disease progression or death (hazard ratio, 0.70) in the bevacizumab group as compared with the placebo group. To control for type I errors in testing for the coprimary end points by means of the log-rank test, ${ }^{21}$ the threshold for statistical significance was set at a two-sided $P$ value of 0.046 for overall survival and 0.004 for progression-free survival. The enrollment goal was 612 eligible patients, and a definitive analysis would be performed after 390 deaths had occurred. Interim monitoring with early stopping criteria for efficacy and futility was performed, as described in the study protocol, and was overseen by the RTOG data and safety monitoring committee.

We used the Kaplan-Meier method to estimate survival distributions and a Cox proportionalhazards model to calculate hazard ratios. ${ }^{22,23}$ To determine whether a molecularly defined subgroup had a selective survival benefit from the addition of bevacizumab to standard treatment, we performed protocol-specified subset analyses for each tumor molecular factor and for combinations of molecular profile and MGMT status. We used the Cox model to perform additional analyses that examined the effects of these factors and recursive partitioning analysis (RPA) class, ${ }^{13}$ a compilation of clinical factors that define a patient's prognosis, with classes ranging from I to VI and higher classes indicating a worse prognosis. This study enrolled patients in RPA classes III, IV, and V. For all these analyses, we used a likelihood-ratio test to evaluate differential treatment effects (interactions). We evaluated the proportionality of hazards using a test based on model residuals and smoothed hazard plots. ${ }^{24,25}$

In the NCB substudy, ${ }^{18}$ we assessed net clinical benefits to determine whether there were differences in changes between the two study groups from baseline to week 46 in patient-reported outcomes (on the basis of the MDASI-BT and EORTC QLQ-C30/BN20) or neurocognitive function (HVLT-R, TMT, and COWA). As specified in the trial protocol, these analyses were restricted to patients who were deemed to be progression-free at the time of the assessment. General linear models were used for longitudinal assessments, with fixed effects for study group and time factors and inclusion of MGMT status and RPA class to adjust for prognostic status. A treatment-by-time interaction effect was added to the model to determine whether there were between-group differences in patterns of response over time, with a $\mathrm{P}$ value of 0.05 considered to indicate statistical significance.

\section{RESULTS}

\section{STUDY PATIENTS}

From April 2009 through May 2011, we enrolled 978 patients, of whom 341 were deemed to be ineligible or otherwise unable to undergo randomization (Fig. 1). Patients who underwent randomization had clinical characteristics that were similar to those who did not undergo randomization (Table S2 in the Supplementary Appendix). A total of 637 patients ( $65 \%$ of those who were enrolled and $96 \%$ of those who were deemed to be eligible) were randomly assigned to receive either placebo (317 patients) or bevacizumab (320 patients); 621 patients were included in the final analysis. One patient who was found to have disease progression before randomization was not included in the analysis of progression-free survival. Baseline characteristics were well balanced between the two study groups (Table S3 in the Supplementary Appendix). The characteristics of patients who con- 


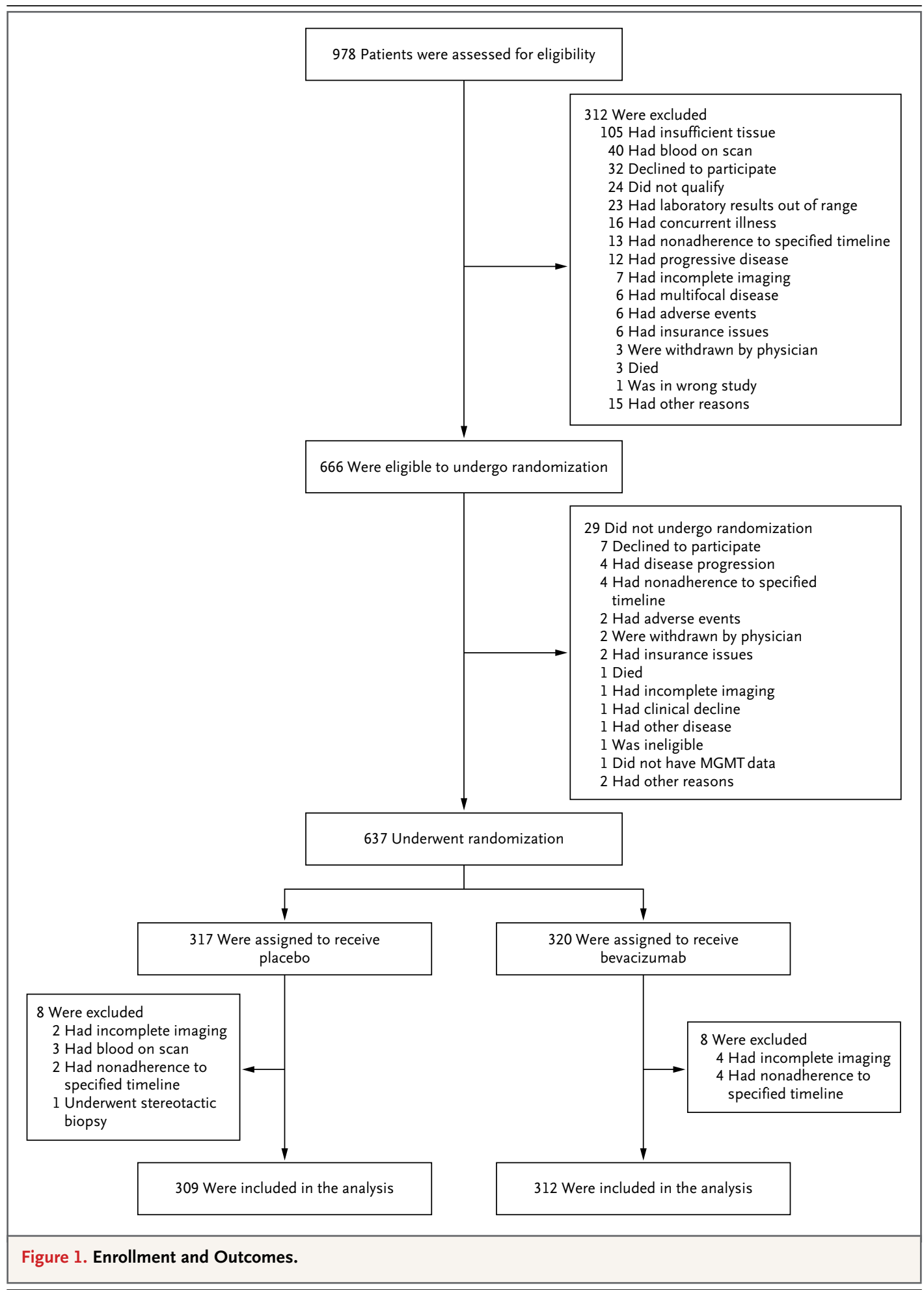

sented to participate in the NCB substudy did not differ from the characteristics of those who were unwilling or unable to participate (Table S4 in the Supplementary Appendix). More than $80 \%$ of the patients who underwent randomization consented to participate in the assessment of net clinical benefits. Only 20 patients could not participate because of native language constraints 
(Table S5 in the Supplementary Appendix). The rate of completion of the test instruments remained high from baseline to week 46 (Table S7 in the Supplementary Appendix).

\section{STUDY TREATMENT}

In the bevacizumab group, 163 of 312 patients (52.2\%) received at least 6 cycles of treatment (median number, 6). In the placebo group, 110 of 309 patients (35.6\%) received at least 6 cycles of maintenance treatment (median number, 3). The full 12 cycles were administered in 57 patients (18.3\%) in the bevacizumab group and in 59 patients $(19.1 \%)$ in the placebo group. Tumor progression or death prompted treatment cessation in 73 of 268 patients $(27.2 \%)$ in the bevacizumab group and in 135 of 274 patients (49.3\%) in the placebo group. Toxicity or intercurrent illness resulted in treatment cessation in 88 patients (32.8\%) in the bevacizumab group and in 45 patients $(16.4 \%)$ in the placebo group.

At the time of this analysis, the treatment assignment had been revealed for 155 of 320 patients (48.4\%) in the bevacizumab group and 178 of 317 patients (56.2\%) in the placebo group. Among patients with disease progression, salvage treatment was planned for 87 of 155 patients $(56.1 \%)$ in the bevacizumab group and 128 of 178 patients $(71.9 \%)$ in the placebo group. Among these patients, the protocol-related bevacizumab regimen was continued in 39 patients $(25.2 \%)$ in the bevacizumab group and was started in 86 patients $(48.3 \%)$ in the placebo group.

\section{TREATMENT OUTCOMES}

\section{Primary Analysis}

At the time of analysis (December 2012), 208 of the 621 patients who were included in the primary analysis (33.5\%) were still alive, with a median follow-up time of 20.5 months. The median overall survival was 15.7 months $(95 \%$ confidence interval [CI], 14.2 to 16.8 ) in the bevacizumab group and 16.1 months (95\% CI, 14.8 to 18.7 ) in the placebo group (hazard ratio for death in the bevacizumab group, 1.13; 95\% CI, 0.93 to 1.37; $\mathrm{P}=0.21$ by the log-rank test) (Fig. $2 \mathrm{~A}$ ).

Tumor progression or death occurred in 512 patients $(82.4 \%)$. The median duration of progression-free survival was 10.7 months (95\% CI, 10.0 to 12.2$)$ in the bevacizumab group as compared with 7.3 months $(95 \% \mathrm{CI}$, 5.9 to 7.9) in the placebo group (hazard ratio for progression or death, $0.79 ; 95 \%$ CI, 0.66 to $0.94 ; \mathrm{P}=0.007$ by the log-rank test) (Fig. 2B). The treatment effect for progression-free survival varied over time $(\mathrm{P}<0.001$ for proportionality of hazards).

\section{MGMT Status, Molecular Profile, and RPA Class}

MGMT status was prognostic regardless of the study treatment. The median overall survival was 14.3 months (95\% CI, 13.6 to 15.3 ) for patients with MGMT unmethylated tumors as compared with 23.2 months (95\% CI, 20.1 to 28.3) for those with methylated tumors (hazard ratio for death in patients with unmethylated tumors, 2.10; $95 \%$ CI, 1.65 to 2.68 ; $\mathrm{P}<0.001)$. The median progression-free survival was 8.2 months $(95 \% \mathrm{CI}$, 7.5 to 9.2) for patients with MGMT unmethylated tumors and 14.1 months (95\% CI, 10.5 to 16.1) for those with methylated tumors (hazard ratio, 1.67; 95\% CI, 1.36 to 2.05; $\mathrm{P}<0.001$ ).

The nine-gene assay, which was developed in a separate trial involving more than 500 patients with glioblastoma who did not receive bevacizumab, was not prognostic in either study group on the basis of a specific favorable or unfavorable categorization of continuous data and a uniform setting of analytic specifications.

The RPA class was prognostic regardless of the study treatment. The median duration of overall survival was 20.1 months (95\% CI, 16.7 to 35.7 ) for patients in class III, 15.6 months $(95 \% \mathrm{CI}$, 14.6 to 17.2) for those in class IV, and 13.2 months (95\% CI, 10.2 to 14.6) for those in class V $(\mathrm{P}<0.001)$. The median progression-free survival was 12.5 months ( $95 \%$ CI, 9.1 to 16.6) for patients in class III, 9.6 months (95\% CI, 8.5 to 10.4) for those in class IV, and 7.1 months (95\% CI, 5.3 to 8.7) for those in class $V(P=0.001)$. Subset analyses that were based on the individual molecular components as well as various combinations of MGMT status and molecular profile were performed. No subset had improved survival with the addition of bevacizumab. These treatment effects were similar across RPA classes (Table 1 ).

Similarly, treatment effects after adjustment for MGMT status, molecular profile, and RPA class were unchanged: hazard ratio for death, 1.12 (95\% CI, 0.91 to 1.37; $\mathrm{P}=0.30$ ); and hazard ratio for disease progression or death, 0.80 ( $95 \% \mathrm{CI}, 0.66$ to $0.97 ; \mathrm{P}=0.02$ ). Testing for statistical interaction did not identify a significant differential effect of treatment according to combinations of MGMT status and molecular profiles or those factors individually. 


\section{NET CLINICAL BENEFITS}

In the NCB substudy, we found greater deterioration over time in the bevacizumab group than in the placebo group on the basis of the between-group difference in the composite scores on the neurocognitive-function test battery $(\mathrm{P}=0.05)$, as well as the scores for the Controlled Oral Word Association Test $(\mathrm{P}=0.003)$ and the Trail Making Test, Part A $(\mathrm{P}=0.04)$. Longitudinal evaluation also revealed greater deterioration in the bevacizumab group on the basis of the MDASI-BT composite symptom score $(\mathrm{P}=0.02)$, composite symptom-interference score $(\mathrm{P}<0.001)$, and the scores for activity-related symptom interference $(\mathrm{P}=0.004)$, mood-related symptom interference $(\mathrm{P}<0.001)$, affective factors $(\mathrm{P}=0.04)$, cognitive factors $(\mathrm{P}=0.01)$, treatment factors $(\mathrm{P}=0.03)$, and generalized or disease factors $(\mathrm{P}=0.01)$, as well as EORTC QLQ-C30/BN20 scores for cognitive functioning ( $\mathrm{P}=0.008)$, motor dysfunction $(\mathrm{P}=0.02)$, and communication deficit $(\mathrm{P}=0.003)$ (Table $\mathrm{S} 8$ in the Supplementary Appendix).

\section{SAFETY AND TOXICITY}

Safety and toxicity data were available for 300 patients in the placebo group and 303 patients in the bevacizumab group. During chemoradiotherapy with bevacizumab or placebo, serious lymphopenia was the most common toxic effect, occurring in approximately $10 \%$ of patients in the two study groups but without clinically significant opportunistic infection. Serious neutropenia was more common in the bevacizumab group than in the placebo group ( $7.3 \%$ vs. $3.7 \%$ ), as was serious thrombocytopenia (10.2\% vs. $7.7 \%$ ) (Table 2 ).

During the maintenance phase, serious adverse events were more prevalent in the bevacizumab group than in the placebo group, including hypertension $(4.2 \%$ vs. $0.9 \%)$, thromboembolic disease ( $7.7 \%$ vs. $4.7 \%)$, wound dehiscence ( $1.5 \%$ vs. $0.9 \%)$, fatigue $(13.1 \%$ vs. $9.0 \%)$, visceral perforation $(1.2 \%$ vs. $0.4 \%)$, and serious hemorrhage (1.5\% vs. $0.9 \%)$. Serious neutropenia was more common in the bevacizumab group ( $10.0 \%$ vs. $5.1 \%$ ), but thrombocytopenia was slightly less common (11.1\% vs. 11.7\%) (Table 2).

\section{DISCUSSION}

Two phase 2 studies have evaluated the use of bevacizumab in patients with newly diagnosed glioblastoma. Vredenburgh and colleagues treat-

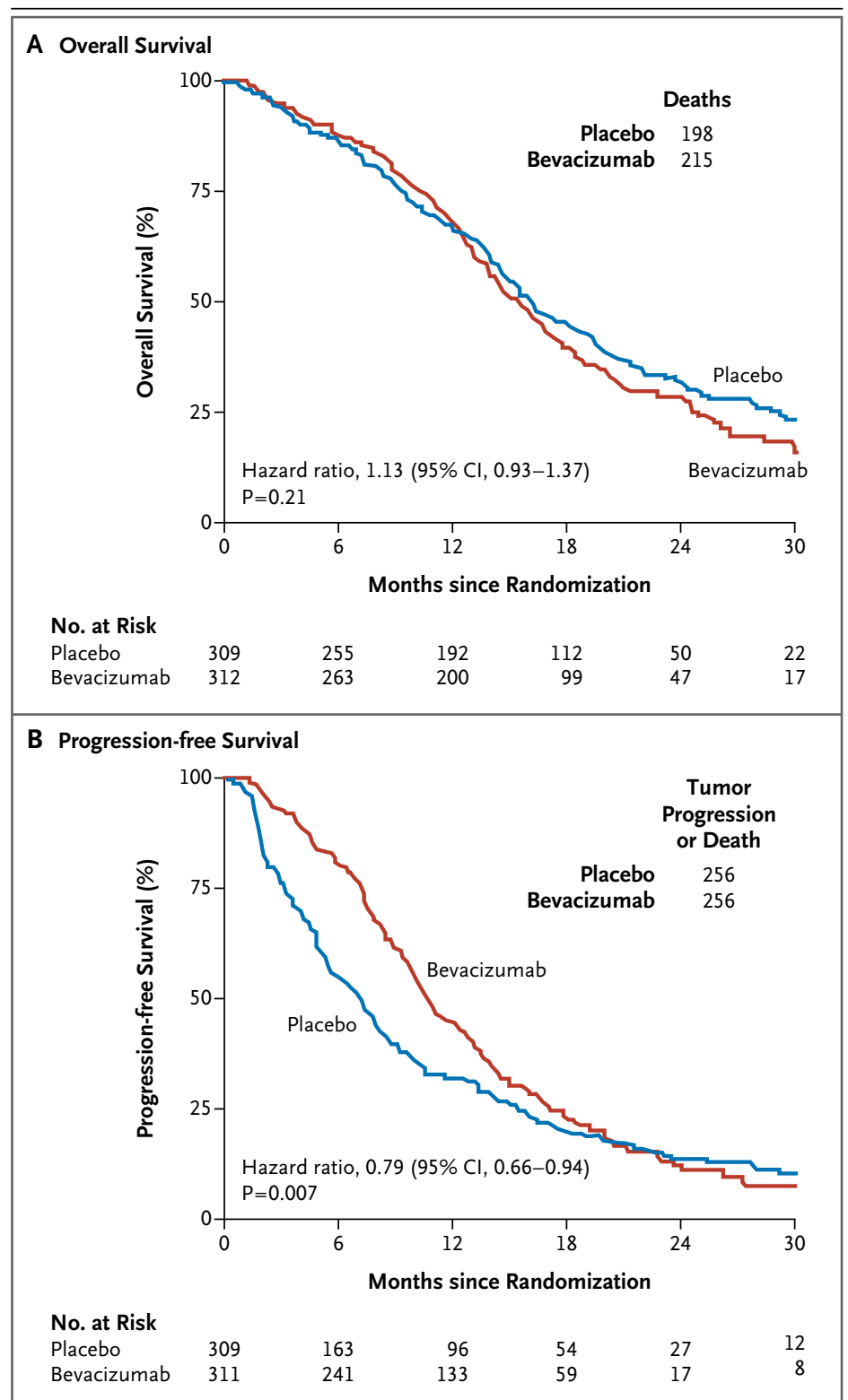

Figure 2. Primary End Points, According to Study Group.

The median rates of overall survival were similar in the bevacizumab and placebo groups (Panel A). The median rate of progression-free survival was higher in the bevacizumab group than in the placebo group but did not reach the prespecified threshold for significance $(P<0.004)$ (Panel $B)$.

ed 75 patients with radiotherapy, temozolomide, and bevacizumab, followed by adjuvant temozolomide, irinotecan, and bevacizumab. ${ }^{26}$ The median overall survival was 21.2 months, which compared favorably with survival in historical controls. These results were very similar to those reported by Lai and collaborators, but they compared results in the study patients with those in a contemporary group of patients who received 


\begin{tabular}{|c|c|c|c|c|}
\hline \multirow[t]{2}{*}{ End Point and Subgroup } & Bevacizumab & Placebo & $\begin{array}{c}\text { Hazard Ratio } \\
(95 \% \mathrm{Cl}) \dagger\end{array}$ & P Value \\
\hline & \multicolumn{2}{|c|}{ mo } & & \\
\hline \multicolumn{5}{|l|}{ All patients } \\
\hline Median overall survival & 15.7 & 16.1 & $1.13(0.93-1.30)$ & 0.21 \\
\hline Median progression-free survival & 10.7 & 7.3 & $0.79(0.66-0.94)$ & 0.007 \\
\hline \multicolumn{5}{|l|}{ MGMT status and molecular profile } \\
\hline \multicolumn{5}{|l|}{ Methylated MGMT } \\
\hline \multicolumn{5}{|l|}{ Favorable molecular profile } \\
\hline Median overall survival & 16.7 & 25.0 & $2.27(0.91-5.68)$ & 0.07 \\
\hline Median progression-free survival & 13.0 & 13.5 & $1.39(0.67-2.89)$ & 0.38 \\
\hline \multicolumn{5}{|l|}{ Unfavorable molecular profile } \\
\hline Median overall survival & 21.1 & 25.3 & $1.24(0.73-2.12)$ & 0.43 \\
\hline Median progression-free survival & 16.9 & 8.4 & $0.63(0.40-0.98)$ & 0.04 \\
\hline \multicolumn{5}{|l|}{ Unmethylated MGMT } \\
\hline \multicolumn{5}{|l|}{ Favorable molecular profile } \\
\hline Median overall survival & 13.9 & 14.6 & $1.02(0.66-1.57)$ & 0.94 \\
\hline Median progression-free survival & 10.1 & 7.3 & $0.72(0.48-1.07)$ & 0.10 \\
\hline \multicolumn{5}{|l|}{ Unfavorable molecular profile } \\
\hline Median overall survival & 14.0 & 14.6 & $1.13(0.86-1.49)$ & 0.36 \\
\hline Median progression-free survival & 9.8 & 5.4 & $0.86(0.67-1.11)$ & 0.25 \\
\hline \multicolumn{5}{|l|}{ RPA class } \\
\hline \multicolumn{5}{|l|}{ III } \\
\hline Median overall survival & 20.6 & 19.8 & $0.98(0.54-1.81)$ & 0.96 \\
\hline Median progression-free survival & 14.9 & 9.5 & $0.74(0.43-1.25)$ & 0.25 \\
\hline \multicolumn{5}{|l|}{ IV } \\
\hline Median overall survival & 15.7 & 15.6 & $1.14(0.90-1.44)$ & 0.29 \\
\hline Median progression-free survival & 10.8 & 7.3 & $0.78(0.63-0.96)$ & 0.02 \\
\hline \multicolumn{5}{|l|}{ V } \\
\hline Median overall survival & 12.6 & 13.3 & $1.01(0.66-1.56)$ & 0.96 \\
\hline Median progression-free survival & 9.8 & 4.4 & $0.70(0.46-1.06)$ & 0.10 \\
\hline
\end{tabular}

* The recursive-partitioning analysis (RPA) classes are as follows: class III: an age of less than 50 years and a Karnofsky performance score of 90 or more (on a scale of 0 to 100, with higher scores indicating better function); class IV, an age of less than 50 years and a Karnofsky performance score of less than 90 (or an age of 50 years or more, a Karnofsky performance score of 70 or more, a gross total or partial tumor resection, and an ability to work); class $V$, an age of 50 years or more, a Karnofsky performance score of 70 or more, a gross total or partial tumor resection, and an inability to work (or an age of 50 years or more, a Karnofsky performance score of 70 or more, and tumor-biopsy specimen only; or an age of 50 years or more and a Karnofsky performance score of less than 70$)$. Cl denotes confidence interval.

$\uparrow$ Hazard ratios are for death (for overall survival) and tumor progression or death (for progression-free survival) in the bevacizumab group, as compared with the placebo group.

standard temozolomide and radiotherapy, which showed an improvement in progression-free survival with bevacizumab (13.6 vs. 7.6 months) but not in overall survival (19.6 vs. 21.1 months). ${ }^{27}$
Our study was designed to determine whether adding bevacizumab to the first-line treatment for glioblastoma would improve patient outcomes. We chose the coprimary end points, overall surviv- 


\begin{tabular}{|c|c|c|c|c|c|c|c|c|}
\hline \multirow[t]{4}{*}{ Event } & \multicolumn{4}{|c|}{ During Chemoradiotherapy } & \multicolumn{4}{|c|}{ During Adjuvant Treatment } \\
\hline & \multicolumn{2}{|c|}{$\begin{array}{l}\text { Bevacizumab } \\
\quad(N=303)\end{array}$} & \multicolumn{2}{|c|}{$\begin{array}{c}\text { Placebo } \\
(\mathrm{N}=300)\end{array}$} & \multicolumn{2}{|c|}{$\begin{array}{l}\text { Bevacizumab } \\
\quad(N=260)\end{array}$} & \multicolumn{2}{|c|}{$\begin{array}{c}\text { Placebo } \\
(\mathrm{N}=233)\end{array}$} \\
\hline & Grade 3 & Grade 4 & Grade 3 & Grade 4 & Grade 3 & Grade 4 & Grade 3 & Grade 4 \\
\hline & \multicolumn{8}{|c|}{ number of patients (percent) } \\
\hline Anemia & $1(0.3)$ & $1(0.3)$ & $1(0.3)$ & 0 & $4(1.5)$ & $2(0.8)$ & $3(1.3)$ & 0 \\
\hline Leukopenia & $10(3.3)$ & $6(2.0)$ & $4(1.3)$ & $3(1.0)$ & $20(7.7)$ & $2(0.8)$ & $14(6.0)$ & 0 \\
\hline Neutropenia & $7(2.3)$ & $15(5.0)$ & $5(1.7)$ & $6(2.0)$ & $20(7.7)$ & $6(2.3)$ & $7(3.0)$ & $5(2.1)$ \\
\hline Lymphopenia & $24(7.9)$ & $8(2.6)$ & $22(7.3)$ & $5(1.7)$ & $27(10.4)$ & $7(2.7)$ & $23(10)$ & $8(3.4)$ \\
\hline Thrombocytopenia & $10(3.3)$ & $21(6.9)$ & $17(5.7)$ & $6(2.0)$ & $18(6.9)$ & $11(4.2)$ & $23(10)$ & $4(1.7)$ \\
\hline Fatigue & $7(2.3)$ & 0 & $8(2.7)$ & 0 & $32(12.3)$ & $2(0.8)$ & $21(9.0)$ & 0 \\
\hline Nausea and vomiting & $2(0.7)$ & 0 & $1(0.3)$ & 0 & $11(4.2)$ & 0 & $4(1.7)$ & 0 \\
\hline Wound dehiscence & $3(1.0)$ & 0 & $1(0.3)$ & 0 & $3(1.2)$ & $1(0.4)$ & $2(0.9)$ & 0 \\
\hline Hypertension & $4(1.3)$ & 0 & $1(0.3)$ & 0 & $11(4.2)$ & 0 & $2(0.9)$ & 0 \\
\hline $\begin{array}{l}\text { Thromboembolic } \\
\text { disease }\end{array}$ & $6(2.0)$ & $8(2.6)$ & $3(1.0)$ & $\begin{array}{c}8(2.7) \text { and } \\
1 \text { grade } 5 \\
(0.3)\end{array}$ & $11(4.2)$ & $\begin{array}{c}8(3.1) \text { and } \\
1 \text { grade } 5 \\
(0.4)\end{array}$ & $7(3.0)$ & $4(1.7)$ \\
\hline Hemorrhage & 0 & 0 & $1(0.3)$ & 0 & $3(1.2)$ & 1 grade $5(0.4)$ & $2(0.9)$ & 0 \\
\hline Visceral perforation & $1(0.3)$ & 0 & 0 & $1(0.3)$ & $2(0.8)$ & $1(0.4)$ & $1(0.4)$ & 0 \\
\hline
\end{tabular}

al and progression-free survival, because of the crossover design of the study. At the time of disease progression, the treatment assignment in the bevacizumab group could be revealed and patients could begin or continue a regimen containing bevacizumab. Therefore, the end point of overall survival was used to determine whether first-line use of bevacizumab was superior to use as a salvage regimen, and the end point of progression-free survival was used to evaluate the potential early benefits of combined treatment.

We found no benefit in overall survival with early administration of bevacizumab. Progressionfree survival was prolonged for the patients receiving first-line bevacizumab, but the hazard ratio $(0.79)$ and $P$ value $(0.007)$ did not reach the predefined criteria. However, we did not assess the efficacy of first-line treatment with bevacizumab in patients with unresected tumors, since the requirement for a tumor specimen excluded patients who had undergone only a diagnostic biopsy. In additional analyses of subgroups of patients defined prospectively on the basis of molecular markers, we did not identify a subgroup of patients who had a selected survival benefit from the early administration of bevacizumab. Further investigations of molecularly defined subgroups may uncover a predictive marker panel for bevacizumab, which would require additional prospective testing.

A similar study, Avastin [bevacizumab] in Glioblastoma (AVAGlio), which also evaluated the role of bevacizumab as first-line treatment in patients with newly diagnosed glioblastoma, was recently completed. ${ }^{28}$ That study showed a result for overall survival that was similar to our finding but showed a different result for progression-free survival on the basis of a statistical design that included a different prespecified alpha level for progression $(\mathrm{P}<0.01)$. The AVAGlio study also evaluated patient-reported outcomes but restricted those data primarily to health-related quality-of-life measures, whereas we also collected measures of symptom burden and interference and the results of objective tests of neurocognitive function.

In our study, we evaluated longitudinal changes in both objective and patient-reported neurocognitive function and in self-reported quality of life and other symptoms in patients who were deemed to be progression-free. These assessments consistently showed that patients receiving bevacizumab, as compared with placebo, had greater deteriora- 
tion in scores on objective tests of neurocognitive function as well as in perceived cognitive function, suggesting either unrecognized tumor progression or bevacizumab-related neurotoxicity.29,30 In addition, among patients without tumor progression on imaging studies, those who were initially treated with bevacizumab reported greater deterioration in the perceived severity of their symptoms, as assessed on the basis of both patient-reported outcomes and symptom-associated interference with daily activities.

In conclusion, we did not observe an overall survival advantage with first-line use of bevacizumab in patients with newly diagnosed glioblastoma. Furthermore, higher rates of neurocog- nitive decline, increased symptom severity, and decline in health-related quality of life were found over time among patients who were treated with bevacizumab.

Supported by grants (U10 CA21661 and U10 CA37422) from the National Cancer Institute and by an unrestricted educational grant from Genentech.

Presented in part at the 2013 Annual Meeting of the American Society of Clinical Oncology, Chicago, May 31-June 4, 2013.

Disclosure forms provided by the authors are available with the full text of this article at NEJM.org.

We thank Kathryn Okrent, Meihua Wang, Sandrine Geinoz, Sharon Hartson, Treena Trotman, Juliet Obropta, and Barbara Kaiser at the Radiation Therapy Oncology Group Headquarters for their efforts in developing and managing the clinical trial; Drs. Andrew Lassman and Jennifer Moughan for their review of a previous draft of the manuscript; and all the site investigators and research staff for their participation.

REFERENCES

1. Stupp R, Mason WP, van den Bent MJ, et al. Radiotherapy plus concomitant and adjuvant temozolomide for glioblastoma. N Engl J Med 2005;352:987-96.

2. Lacroix M, Abi-Said D, Fourney DR, et al. A multivariate analysis of 416 patients with glioblastoma multiforme: prognosis, extent of resection, and survival. J Neurosurg 2001;95:190-8.

3. Stupp R, Hegi ME, Mason WP, et al. Effects of radiotherapy with concomitant and adjuvant temozolomide versus radiotherapy alone on survival in glioblastoma in a randomised phase III study: 5-year analysis of the EORTC-NCIC trial. Lancet Oncol 2009;10:459-66.

4. Lund EL, Spang-Thomsen M, SkovgaardPoulsen H, Kristjansen PE. Tumor angiogenesis - a new therapeutic target in gliomas. Acta Neurol Scand 1998;97:52-62.

5. Jain RK, di Tomaso E, Duda DG, Loeffler JS, Sorensen AG, Batchelor TT. Angiogenesis in brain tumours. Nat Rev Neurosci 2007;8:610-22.

6. Batchelor TT, Mulholland P, Neyns B, et al. Phase III randomized trial comparing the efficacy of cediranib as monotherapy, and in combination with lomustine, versus lomustine alone in patients with recurrent glioblastoma. J Clin Oncol 2013;31: 3212-8.

7. Lee EQ, Kuhn J, Lamborn KR, et al. Phase I/II study of sorafenib in combination with temsirolimus for recurrent glioblastoma or gliosarcoma: North American Brain Tumor Consortium study 05-02. Neuro Oncol 2012;14:1511-8.

8. Ferrara N, Hillan KJ, Novotny W. Bevacizumab (Avastin), a humanized anti-VEGF monoclonal antibody for cancer therapy. Biochem Biophys Res Commun 2005;333: 328-35.

9. Friedman HS, Prados MD, Wen PY, et al. Bevacizumab alone and in combination with irinotecan in recurrent glioblastoma. J Clin Oncol 2009;27:4733-40.
10. Kreisl TN, Kim L, Moore K, et al. Phase II trial of single-agent bevacizumab followed by bevacizumab plus irinotecan at tumor progression in recurrent glioblastoma. J Clin Oncol 2009;27:740-5.

11. Jain RK. Normalization of tumor vasculature: an emerging concept in antiangiogenic therapy. Science 2005;307:58-62.

12. Zelen $M$. The randomization and stratification of patients to clinical trials. J Chronic Dis 1974;27:365-75.

13. Colman H, Zhang L, Sulman EP, et al. A multigene predictor of outcome in glioblastoma. Neuro Oncol 2010;12:49-57.

14. Vlassenbroeck I, Califice S, Diserens AC, et al. Validation of real-time methylationspecific PCR to determine O6-methylguanine-DNA methyltransferase gene promoter methylation in glioma. J Mol Diagn 2008;10:332-7.

15. Armstrong TS, Mendoza T, Gning I, et al. Validation of the M.D. Anderson Symptom Inventory Brain Tumor Module (MDASI-BT). J Neurooncol 2006;80:27-35.

16. Osoba D, Aaronson NK, Muller M, et al. The development and psychometric validation of a brain cancer quality-of-life questionnaire for use in combination with general cancer-specific questionnaires. Qual Life Res 1996;5:139-50.

17. Wefel JS, Cloughesy T, Zazzali JL, et al. Neurocognitive function in patients with recurrent glioblastoma treated with bevacizumab. Neuro Oncol 2011;13:660-8.

18. Armstrong TS, Wefel JS, Wang M, et al. Net clinical benefit analysis of Radiation Therapy Oncology Group 0525: a phase III trial comparing conventional adjuvant temozolomide with dose-intensive temozolomide in patients with newly diagnosed glioblastoma. J Clin Oncol 2013; 31:4076-84.

19. Macdonald DR, Cascino TL, Schold SC Jr, Cairncross JG. Response criteria for phase II studies of supratentorial malignant glioma. J Clin Oncol 1990;8:1277-80.
20. Sanghera P, Perry J, Sahgal A, et al. Pseudoprogression following chemoradiotherapy for glioblastoma multiforme. Can J Neurol Sci 2010;37:36-42.

21. Mantel N. Evaluation of survival data and two new rank order statistics arising in its consideration. Cancer Chemother Rep 1966;50:163-70

22. Kaplan EL, Meier P. Nonparametric estimation from incomplete observations. J Am Stat Assoc 1958;53:457-81.

23. Cox DR. Regression models and lifetables. J R Stat Soc B 1972;34:187-202. 24. Grambsch P, Louis TA, Bostick RM, et al. Statistical analysis of proliferative index data in clinical trials. Stat Med 1994;13:1619-34.

25. Müller HG, Wang JL. Hazard rate estimation under random censoring with varying kernels and bandwidths. Biometrics 1994;50:61-76

26. Vredenburgh JJ, Desjardins A, Reardon $\mathrm{DA}$, et al. The addition of bevacizumab to standard radiation therapy and temozolomide followed by bevacizumab, temozolomide, and irinotecan for newly diagnosed glioblastoma. Clin Cancer Res 2011;17: 4119-24.

27. Lai A, Tran A, Nghiemphu PL, et al. Phase II study of bevacizumab plus temozolomide during and after radiation therapy for patients with newly diagnosed glioblastoma multiforme. J Clin Oncol 2011;29:142-8

28. Chinot OL, Wick W, Mason W, et al. Bevacizumab plus radiotherapy-temozolomide for newly diagnosed glioblastoma. N Engl J Med 2014;370:709-22.

29. Sorensen AG, Batchelor TT, Wen PY, Zhang WT, Jain RK. Response criteria for glioma. Nat Clin Pract Oncol 2008;5: 634-44.

30. Mackenzie F, Ruhrberg C. Diverse roles for VEGF-A in the nervous system. Development 2012;139:1371-80.

Copyright (๑) 2014 Massachusetts Medical Society. 